\title{
PAPER
}

\section{Cognitive and behavioural effects of chronic stimulation of the subthalamic nucleus in patients with Parkinson's disease}

\author{
A Daniele, A Albanese, M F Contarino, P Zinzi, A Barbier, F Gasparini, L M A Romito, \\ A R Bentivoglio, M Scerrati
}

See end of article for authors' affiliations

Correspondence to:

Dr Antonio Daniele, Istituto di Neurologia, Università

Cattolica, Largo A Gemelli 8, 1-00168 Rome, Italy; adaniele@rm.unicatt.it

Received

25 January 2002

In final revised form

8 November 2002

Accepted

11 November 2002

\begin{abstract}
Objective: To investigate cognitive and behavioural effects of bilateral lead implants for high frequency stimulation (HFS) of the subthalamic nucleus in patients with Parkinson's disease; and to discriminate between HFS and the effects of surgical intervention on cognitive function by carrying out postoperative cognitive assessments with the stimulators turned on or off.

Methods: Motor, cognitive, behavioural, and functional assessments were undertaken in 20 patients with Parkinson's disease before implantation and then at three, six, and 12 months afterwards. Nine patients were also examined 18 months after surgery. Postoperative cognitive assessments were carried out with stimulators turned off at three and 18 months, and turned on at six and 12 months.

Results: Cognitive assessment showed a significant postoperative decline in performance on tasks of letter verbal fluency (across all postoperative assessments, but more pronounced at three months) and episodic verbal memory (only at three months, with stimulators off). At three, six, and 12 months after surgery, there was a significant improvement in the mini-mental state examination and in a task of executive function (modified Wisconsin card sorting test). On all postoperative assessments, there was an improvement in parkinsonian motor symptoms, quality of life, and activities of daily living while off antiparkinsonian drugs. A significant postoperative decrease in depressive and anxiety symptoms was observed across all assessments. Similar results were seen in the subgroup of nine patients with an 18 month follow up. Following implantation, three patients developed transient manic symptoms and one showed persistent psychic akinesia.

Conclusions: Bilateral HFS of the subthalamic nucleus is a relatively safe procedure with respect to long term cognitive and behavioural morbidity, although individual variability in postoperative cognitive and behavioural outcome invites caution. Stimulation of the subthalamic nucleus does not per se appear to impair cognitive performance in patients with Parkinson's disease and may alleviate the postpoperative decline in verbal fluency.
\end{abstract}

l: $\mathrm{n}$ recent years, various new procedures have been introduced for the treatment of severe Parkinson's disease, including posteroventral pallidotomy and chronic electrical high frequency stimulation (HFS) of the internal globus pallidus or the subthalamic nucleus. This is a reversible and adaptable procedure which may produce marked improvement in the cardinal motor symptoms of Parkinson's disease, through a functional inactivation of the internal globus pallidus or the subthalamic nucleus. ${ }^{1-4}$ However, the effects of these HFS procedures on cognition and behaviour in Parkinson's disease have still to be clearly defined. They might give rise to cognitive and behavioural changes either by inducing surgical microlesions or by modifying the activity of the basal ganglia-thalamocortical circuitry, with possible additional effects on distant neural systems. The ventromedial part of the subthalamic nucleus is linked to associative cortical areas and may play a role in cognition and mood.

Cognition has been assessed in various studies of patients with Parkinson's disease before and after surgery for HFS of the subthalamic nucleus, with stimulators turned on (on condition) during the postoperative evaluation. These studies, however, have been unable to distinguish between the cognitive effects of the surgical intervention and those of HFS per se, because postoperative cognitive assessments were systematically carried out only with stimulators on. In all the studies that have assessed cognition in patients with Parkinson's disease before and after subthalamic nucleus HFS, and with stimulators on during the postoperative evaluation, there has been a worsening in verbal fluency tasks after the procedure. In addition, a decline in tasks sensitive to frontal lobe dysfunction and in memory tasks has been reported in some studies $^{5-7}$ but not in others. ${ }^{8}$ Postoperative cognitive performance in patients treated by HFS with the stimulators on versus off has been assessed in two studies, but there are discrepancies in the findings. ${ }^{910}$ In addition, those studies have only focused on tasks sensitive to frontal lobe dysfunction, and the postoperative cognitive assessments were done in on and off conditions only at short test-retest intervals.

In the present study, we had two main objectives: first, to investigate cognitive and behavioural effects of bilateral HFS of the subthalamic nucleus in a homogeneous group of patients with Parkinson's disease, in order to assess the safety of the procedure and any cognitive morbidity that might be involved; and second, to attempt to discriminate between the effects on cognition of surgical intervention and the effects of HFS of the subthalamic nucleus per se, by doing postoperative cognitive assessments under two different stimulation conditions (stimulators turned on or off). We set out to explore a

Abbreviations: $A D L$, activities of daily living; HFS, high frequency stimulation; LEDD, levodopa equivalent daily dosage; MMSE, mini-mental state examination; MWCST, modified Wisconsin card sorting test; PDQL, Parkinson's disease quality of life questionnaire; RAVLT, Rey's auditory verbal learning test; SAS, Zung self rating anxiety scale; SDS, Zung self rating depression scale; UPDRS, unified Parkinson's disease rating scale 
Table 1 Main demographic and clinical characteristics of patients with Parkinson's disease at the time of the implant

\begin{tabular}{ll}
\hline Number of patients & 20 \\
Sex ratio (male/female) & $11 / 9$ \\
Age (years) & $57.0(7.8)$ \\
Educational level (years) & $11.8(5.2)$ \\
Disease duration (years) & $14.2(5.4)$ \\
Levodopa equivalent daily dose & $1395.8(644.1)$ \\
\hline Values are mean (SD) or $\mathrm{n}$. &
\end{tabular}

wide range of cognitive functions (including episodic memory, working memory, and non-verbal abstract reasoning) in addition to tasks sensitive to frontal lobe dysfunction.

It is well documented that in serial neuropsychological assessments improved performance may result from the repeated administration of a cognitive task (practice effects), especially with short test-retest intervals. ${ }^{11}$ Accordingly, in order to limit the influence of learning from repeated cognitive testing on the comparisons between different stimulation conditions (stimulators on versus off), relatively long intervals between cognitive assessments under different stimulation conditions were chosen in our study.

\section{METHODS}

\section{Patients}

Twenty patients with Parkinson's disease (11 men, nine women) were selected for implantation according to the following inclusion criteria ${ }^{12}$ :

- a diagnosis of Parkinson's disease made according to the UK Parkinson's disease brain bank criteria ${ }^{13}$;

- disabling motor fluctuations with prolonged off periods ( $25 \%$ or more of the waking hours) and on state dyskinesias;

- unsatisfactory management of fluctuations with drug treatment;

- Hoehn and Yahr stage $\geqslant$ III in the practically defined off condition. ${ }^{14}$

Exclusion criteria were a history of previous brain surgery or implantation of a heart pacemaker, an unstable antiparkinsonian drug regimen, severe psychiatric symptoms (hallucinations, major depression), moderate or severe cognitive impairment as measured by scores below 20 on the minimental state examination (MMSE), a diagnosis of dementia according to the Diagnostic and Statistical Manual of Mental Disorders, fourth edition (DSM-IV), ${ }^{15}$ and unsatisfactory general condition.
The main demographic and clinical characteristics of patients at time of implantation are given in table 1 .

\section{Neurosurgical procedures}

All the patients received a bilateral stereotactic subthalamic nucleus implant, following neurosurgical procedures that have already been described. ${ }^{12}$ The mean (SD) voltage of stimulation was $2.64(0.36) \mathrm{V}$ at three months, $2.76(0.33) \mathrm{V}$ at six months, $2.83(0.39)$ V at 12 months, and $2.89(0.45) \mathrm{V}$ at 18 months. Thus, there was a slight increase in the energy delivery required over time in order to assure optimal control of parkinsonian motor symptoms. This was comparable with the setting variations reported in other studies. ${ }^{3}$

\section{Clinical assessment}

All the patients underwent motor, cognitive, behavioural, and functional assessments, both preoperatively and postoperatively. Motor assessment was carried out using the motor examination part of the unified Parkinson's disease rating scale (UPDRS), ${ }^{16}$ with evaluations before surgery and at three, six, 12, and 18 months after the implant, both with and without antiparkinsonian drug treatment. ${ }^{12}$ All cognitive, behavioural, and functional assessments were done while the patients were on anti-parkinsonian drugs, both before and after surgery. Cognitive assessment was carried out by means of a neuropsychological test battery which was assembled according to the following criteria: relatively short duration (two to three hours), assessment of a range of cognitive functions known to be affected by Parkinson's disease, and inclusion of tests known to be sensitive to the effects of pallidotomy. Tests sensitive to motor speed were not included in the neuropsychological battery to minimise the possible deleterious effects of dyskinesias on the results.

Overall cognitive status was assessed by means of the MMSE. ${ }^{17}$ Verbal short term memory was assessed by digit span forward and backward. ${ }^{18}$ Spatial short term memory was evaluated by Corsi's block tapping test. ${ }^{19}$ Episodic verbal memory was assessed by Rey's auditory verbal learning test (RAVLT). ${ }^{20}$ For the RAVLT, randomised parallel forms were used in different assessments to limit practice effects. To investigate non-verbal abstract reasoning we used Raven's progressive matrices ' $47 .^{21}$ To assess frontal-executive functions, we used the modified Wisconsin card sorting test (MWCST) ${ }^{22}$ and a letter verbal fluency task..$^{23}$ The score range for those neuropsychological measures that have a prefixed maximum score is reported in tables 3 and 4 .

Behavioural assessment included an evaluation of mood and anxiety using Zung's self rating depression scale (SDS $)^{24}$ and Zung's self rating anxiety scale (SAS), ${ }^{25}$ which can quantify symptoms of depression and anxiety at the time of testing. In addition, a clinical interview to detect the presence of

Table 2 Motor scores (without and with anti-parkinsonian drug treatment), levodopa equivalent daily dose (LEDD) at baseline and at follow up visits with stimulators turned on

\begin{tabular}{|c|c|c|c|c|c|c|}
\hline & Baseline & 3 Months & 6 Months & 12 Months & Baseline & 18 Months \\
\hline Number of patients & 20 & 20 & 20 & 20 & 9 & 9 \\
\hline UPDRS motor score off drug treatment & $58.8(9.7)$ & $27.3(10.9)$ & $28.8(10.6)$ & $30.9(15.6)$ & $60.8(8.9)$ & $27.0(14.3)$ \\
\hline Change* $^{*}$ & & $-53.6 \%$ & $-51.1 \%$ & $-47.3 \%$ & & $-55.6 \%$ \\
\hline p Value* & & 0.0001 & 0.0001 & 0.0002 & & 0.0101 \\
\hline UPDRS motor score on drug treatment & $24.0(9.0)$ & $25.8(12.3)$ & $19.9(10.8)$ & $22.1(12.3)$ & $25.0(8.0)$ & $17.3(5.5)$ \\
\hline Change* $^{*}$ & & $+7.6 \%$ & $-16.9 \%$ & $-8.1 \%$ & & $-30.7 \%$ \\
\hline p Value* & & NS & NS & NS & & NS \\
\hline LEDD & $1395.8(644.1)$ & $613.6(398.5)$ & $594.2(309.7)$ & $500.7(328.8)$ & $1185.1(426.5)$ & $535.3(458.1)$ \\
\hline Change* & & $-56.0 \%$ & $-57.4 \%$ & $-57.4 \%$ & & $-53.8 \%$ \\
\hline p Value* & & $<0.0001$ & 0.0008 & 0.0002 & & 0.0209 \\
\hline
\end{tabular}

Values are mean (SD) or $\mathrm{n}$ unless specified.

*Compared with baseline.

LEDD, levodopa equivalent daily dose; UPDRS, unified Parkinson's disease rating scale. 


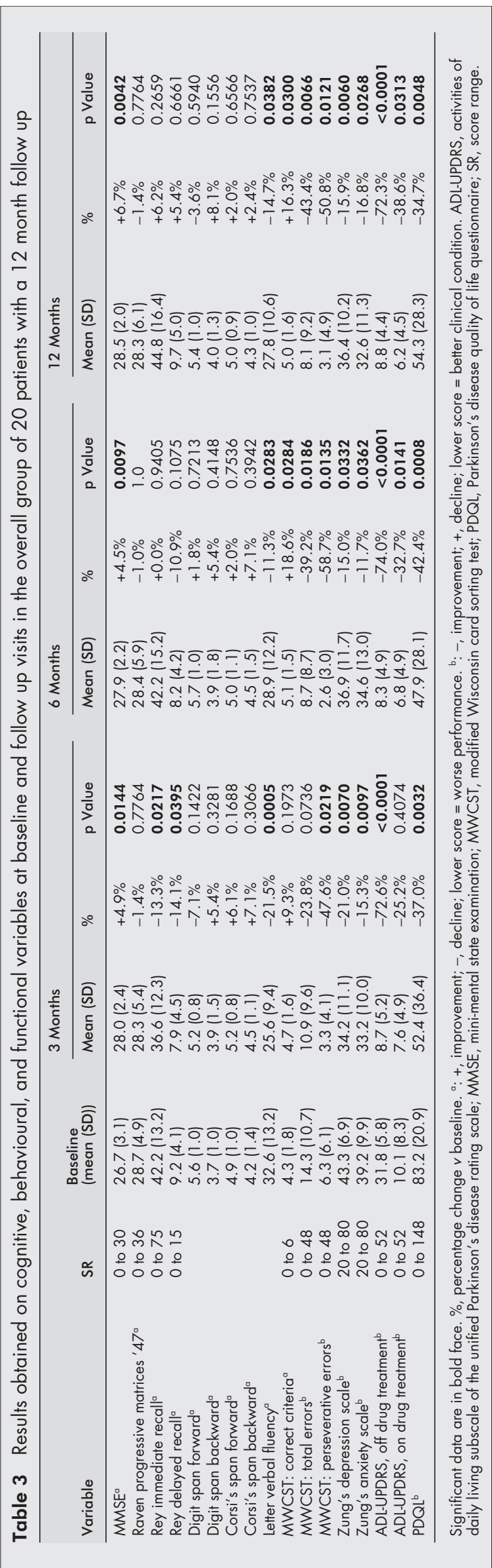

behavioural abnormalities or psychiatric disorders was conducted in all patients.

Functional assessment included evaluation of activities of daily living (ADL) and of quality of life. ADL during periods with and without antiparkinsonian drug treatment in the last month were evaluated using the ADL subscale of the UPDRS. ${ }^{16}$ Quality of life during the last three months was assessed by the Parkinson's disease quality of life questionnaire (PDQL). ${ }^{26}$

\section{Study design}

A preoperative assessment (baseline) was made during the four weeks preceding the bilateral implantation of electrodes. In the overall group of 20 patients, postoperative assessments were carried out at three, six, and 12 months after implantation. In addition, nine of the 20 patients also underwent a postoperative assessment 18 months after implantation. Postoperative assessments of cognitive functioning were done in two different stimulation conditions according to an ABBA design: at three and 18 months the patients were examined 60 minutes after turning the stimulators off, while at six and 12 months the stimulators were left in the on condition. As the repeated administration of a cognitive task (especially with short test-retest intervals) may result in an improved performance from practice, relatively long intervals between cognitive assessments under different stimulation conditions were chosen. ${ }^{11}$

\section{Statistical analyses}

To assess the outcome of the patients as a group (group outcome) and as individuals (individual outcome), two sets of analyses were carried out. To evaluate group outcome, the scores of the sample of patients obtained on each postoperative assessment were compared with preoperative scores by means of the Wilcoxon's signed rank test. This non-parametric test was chosen because of the small sample size and the nonnormally distributed data. Given the explorative nature of our study, the standard non-corrected significance $\alpha$ level of $\mathrm{p}<0.05$ was used to reduce the risk of a type II error. However, given the risk of type I error with multiple comparisons in a relatively small sample of patients, significant results should be accepted with caution when the $\alpha$ levels are only marginally less than $0.05 .{ }^{56}$

In addition, in the overall sample of 20 patients each individual postoperative raw score was transformed to a standard $z$ score, using means and standard deviation values of the sample at baseline, to evaluate postoperative changes among individual patients on cognitive, behavioural, and functional variables. The criterion of an increase of more than $+1.0 \mathrm{SD}$ was used to register individual postoperative improvements, while a decrease of more than -1.0 SD was used to register individual postoperative declines. ${ }^{27}$

\section{RESULTS}

\section{Group outcome}

After surgery, parkinsonian motor symptoms improved in all the patients (table 2). Compared with preoperative scores, there was a significant improvement in UPDRS motor scores in all postoperative assessments without antiparkinsonian drug treatment, and a significant reduction in the total levodopa equivalent daily dose (LEDD). Motor fluctuations improved in all patients and on state dyskinesias were markedly reduced.

The group outcome on cognitive variables for the overall group of 20 patients with a 12 month follow up is reported in table 3. On the letter verbal fluency task, there was a highly significant postoperative decline at three months, with stimulators off (mean decline, $-21.5 \% ; \mathrm{p}=0.0005)$, and a significant decline at six months (mean decline, $-11.3 \% ; \mathrm{p}=0.0283$ ) and 12 months, with stimulators on (mean decline, $-14.8 \%$; 


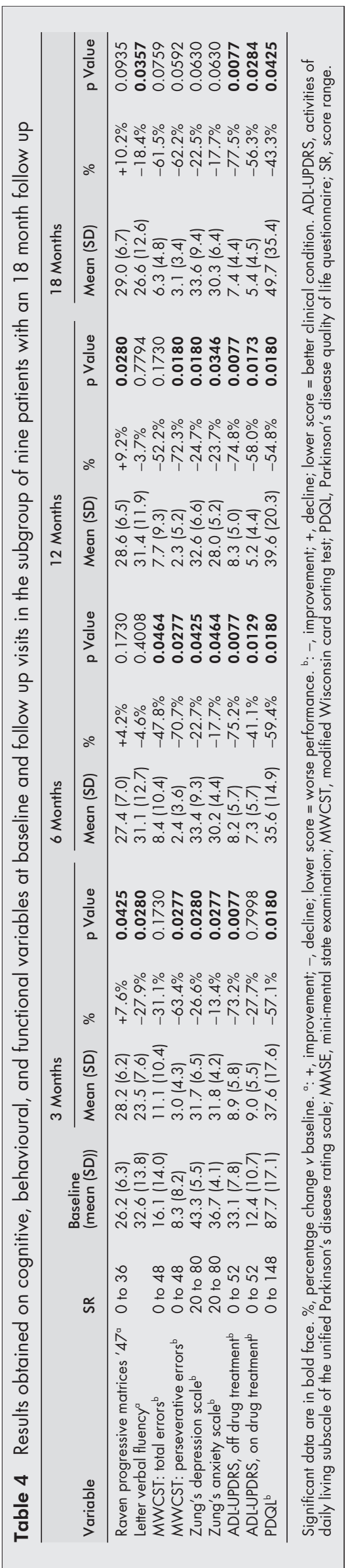

$p=0.0382$ ). On the episodic verbal memory task, a postoperative decline was observed only at three months with stimulators in off state, on both immediate recall (mean decline, $-13.3 \% ; p=0.0217$ ) and delayed recall of the RAVLT (mean decline, $-14.1 \% ; p=0.0395)$. Conversely, an improvement in postoperative performance was detected on the MMSE at three months (mean improvement, $+4.9 \%$; $=0.0144$ ), six months (mean improvement, $+4.5 \% ; \mathrm{p}=0.0097$ ), and 12 months (mean improvement, $+6.7 \% ; \mathrm{p}=0.0042$ ). On the MWCST, there was improved postoperative performance in the number of correct criteria at six months (mean increase, $+18.6 \% ; p=0.0284$ ) and 12 months (mean increase, $+16.3 \%$; $\mathrm{p}=0.0300)$, and in the number of total errors at six months (mean decrease, $-39.2 \% ; p=0.0186$ ) and 12 months (mean decrease, $-43.4 \% ; \mathrm{p}=0.0066)$. On this latter task, a decrease in perseverative errors was observed at three months (mean decrease, $-47.6 \% ; \mathrm{p}=0.0219$ ), six months (mean decrease, $-58.7 \% ; \mathrm{p}=0.0135$ ), and 12 months (mean decrease, $-50.8 \%$; $\mathrm{p}=0.0121$ ).

With respect to the outcome on behavioural and functional variables in the overall group of 20 patients with a 12 month follow up (table 3, lower part), there was a decrease in depressive symptoms at three months (mean decrease on SDS, $-21.0 \% ; p=0.0070$ ), six months (mean decrease on SDS, $-14.8 \% ; \mathrm{p}=0.0332$ ), and 12 months (mean decrease on SDS, $-15.9 \% ; p=0.0060$ ). A decrease in anxiety symptoms was also observed at three months (mean decrease on SAS, $-15.3 \% ; p=0.0097$ ), six months (mean decrease on SAS, $-11.7 \% ; p=0.0362$ ), and 12 months (mean decrease on SAS, $-16.8 \% ; p=0.0268$ ). There was an improvement in the quality of life at three months (mean decrease on PDQL, $-37.0 \%$; $\mathrm{p}=0.0032$ ), six months (mean decrease on PDQL, $-42.4 \%$; $\mathrm{p}=0.0008$ ), and 12 months (mean decrease on PDQL, $-34.7 \% ; p=0.0048$ ). A highly significant improvement in activities of daily living off drug treatment was observed at three months (mean decrease on UPDRS-ADL, -72.6\%; $\mathrm{p}<0.0001$ ), six months (mean decrease on UPDRS-ADL, $-74.0 \% ; \mathrm{p}<0.0001$ ), and 12 months (mean decrease on UPDRS-ADL, $-72.3 \% ; \mathrm{p}<0.0001$ ). For ADL on drug treatment, there was significant improvement only at six months (mean decrease on UPDRS-ADL, $-32.7 \% ; p=0.0141$ ) and 12 months (mean decrease on UPDRS-ADL, $-38.6 \%$; $\mathrm{p}=0.0313)$.

The results on cognitive, behavioural, and functional variables in the subgroup of nine patients with an 18 month follow up were quite similar to those obtained in the overall group of 20 patients, as shown in table 4 . This shows only those tasks with significant changes from baseline. Interestingly, a postoperative decline in letter verbal fluency was observed only at three months (mean decrease, $-27.9 \%$; $\mathrm{p}=0.0280$ ) and 18 months (mean decrease, $-18.4 \%$; $p=0.0357$ ) with stimulators in the off condition, but not at six and 12 months (in the on condition). On Raven's progressive matrices ' 47 , there was an improvement at three months (mean increase, $+7.6 \% ; \mathrm{p}=0.0425$ ) and 12 months (mean increase, $+9.2 \% ; \mathrm{p}=0.0280$ ). Moreover, a significantly improved postoperative performance was observed on the MWCST for both the number of total errors at six months (mean decrease, $-47.8 \% ; \mathrm{p}=0.0464$ ) and the number of perseverative errors at three months (mean decrease, $-63.4 \%$; $\mathrm{p}=0.0277$ ), six months (mean decrease, $-70.7 \%$; $\mathrm{p}=0.0277$ ), and 12 months (mean decrease, $-73.2 \%$; $\mathrm{p}=0.0180$ ).

In this subgroup of nine patients (table 4), an improvement in quality of life and ADL while off antiparkinsonian drugs was observed in all postoperative assessments, while a decrease in both depressive and anxiety symptoms was observed in all postoperative assessments except for that at 18 months. There was also a significant improvement in ADL while on drug treament at six, 12, and 18 months. 
Table 5 Individual postoperative changes of cognitive, behavioural, and functional variables in the 20 patients with a 12 month follow up

\begin{tabular}{|c|c|c|c|c|c|c|c|c|c|}
\hline \multirow[b]{2}{*}{ Variable } & \multicolumn{3}{|c|}{ Improvement (SD >+1) } & \multicolumn{3}{|c|}{ No change (SD +1 to -1 ) } & \multicolumn{3}{|c|}{ Decline (SD $<-1)$} \\
\hline & 3 Months & 6 Months & 12 Months & 3 Months & 6 Months & 12 Months & 3 Months & 6 Months & 12 Months \\
\hline MMSE & $35 \%$ & $25 \%$ & $40 \%$ & $55 \%$ & $75 \%$ & $55 \%$ & $10 \%$ & $0 \%$ & $5 \%$ \\
\hline Raven's progressive matrices ' 47 & $20 \%$ & $25 \%$ & $25 \%$ & $60 \%$ & $45 \%$ & $50 \%$ & $20 \%$ & $30 \%$ & $25 \%$ \\
\hline Rey's immediate recall & $5 \%$ & $20 \%$ & $30 \%$ & $55 \%$ & $55 \%$ & $50 \%$ & $40 \%$ & $25 \%$ & $20 \%$ \\
\hline Rey's delayed recall & $15 \%$ & $15 \%$ & $40 \%$ & $45 \%$ & $60 \%$ & $35 \%$ & $40 \%$ & $25 \%$ & $25 \%$ \\
\hline Digit span forward & $5 \%$ & $15 \%$ & $10 \%$ & $80 \%$ & $75 \%$ & $70 \%$ & $15 \%$ & $10 \%$ & $20 \%$ \\
\hline Digit span backward & $25 \%$ & $40 \%$ & $30 \%$ & $35 \%$ & $25 \%$ & $40 \%$ & $40 \%$ & $35 \%$ & $30 \%$ \\
\hline Corsi's span forward & $45 \%$ & $35 \%$ & $30 \%$ & $50 \%$ & $65 \%$ & $65 \%$ & $5 \%$ & $0 \%$ & $5 \%$ \\
\hline Corsi's span backward & $25 \%$ & $15 \%$ & $10 \%$ & $70 \%$ & $75 \%$ & $85 \%$ & $5 \%$ & $10 \%$ & $5 \%$ \\
\hline Letter verbal fluency & $0 \%$ & $10 \%$ & $0 \%$ & $74 \%$ & $63 \%$ & $79 \%$ & $26 \%$ & $26 \%$ & $21 \%$ \\
\hline MWCST: No of criteria & $0 \%$ & $0 \%$ & $0 \%$ & $83 \%$ & $89 \%$ & $83 \%$ & $17 \%$ & $11 \%$ & $17 \%$ \\
\hline MWCST: total errors & $33 \%$ & $33 \%$ & $39 \%$ & $55 \%$ & $61 \%$ & $50 \%$ & $11 \%$ & $6 \%$ & $11 \%$ \\
\hline MWCST: perseverative errors & $39 \%$ & $33 \%$ & $44 \%$ & $61 \%$ & $67 \%$ & $44 \%$ & $0 \%$ & $0 \%$ & $11 \%$ \\
\hline Zung's depression scale & $76 \%$ & $58 \%$ & $65 \%$ & $12 \%$ & $25 \%$ & $29 \%$ & $12 \%$ & $17 \%$ & $6 \%$ \\
\hline Zung's anxiety scale & $50 \%$ & $37 \%$ & $47 \%$ & $37 \%$ & $50 \%$ & $47 \%$ & $12 \%$ & $12 \%$ & $6 \%$ \\
\hline ADL-UPDRS, off drug treatment & $100 \%$ & $100 \%$ & $100 \%$ & $0 \%$ & $0 \%$ & $0 \%$ & $0 \%$ & $0 \%$ & $0 \%$ \\
\hline ADL-UPDRS, on drug treatment & $0 \%$ & $10 \%$ & $5 \%$ & $95 \%$ & $90 \%$ & $95 \%$ & $5 \%$ & $0 \%$ & $0 \%$ \\
\hline PDQL & $75 \%$ & $75 \%$ & $69 \%$ & $6 \%$ & $19 \%$ & $25 \%$ & $19 \%$ & $6 \%$ & $6 \%$ \\
\hline
\end{tabular}

ADL-UPDRS, activities of daily living subscale of the unified Parkinson's disease rating scale; MMSE, mini-mental state examination; MWCST, modified

Wisconsin card sorting test; PDQL, Parkinson's disease quality of life questionnaire.

\section{Individual outcome}

The individual outcome on cognitive, behavioural, and functional variables on three month, six month, and 12 month postoperative assessments in the sample of 20 patients is given in table 5. After implantation, no patient became demented according to the DSM-IV criteria. ${ }^{15}$

Across all postoperative assessments, the percentage of patients who showed deterioration on the letter verbal fluency task was substantially greater than the percentage who improved. Moreover, on an episodic memory task (immediate and delayed recall of RAVLT) at three months, the percentage of patients who declined was also substantially greater than the percentage who improved. By contrast, across all postoperative assessments the percentage of patients who improved on other cognitive tasks (MMSE, Corsi's span, number of total errors and perseverative errors on the MWCST) was greater than the percentage who declined. A marked degree of individual variability was observed on digit span backward across all postoperative assessments. A minority of patients showed no postoperative change on this verbal short term memory task, while most had either a postoperative improvement or a decline.

At three, six, and 12 months, on most behavioural and functional variables assessing depressive and anxiety symptoms, quality of life, and ADL while off antiparkinsonian drugs, the percentage of patients who showed a postoperative improvement was markedly greater than the percentage with a postoperative decline.

\section{Adverse events}

Eleven of the 20 patients experienced adverse events. Four had persistent complications: psychic akinesia (one patient), mild worsening of dysarthria (two patients), and a mild worsening of hypophonia (one patient). The patient who showed worsening of hypophonia also experienced transient ballistic or choreic dyskinesias of the neck or limbs, as did one of the patients who showed a worsening of dysarthria. Seven further patients had only transient adverse effects: three showed transient manic symptoms with hypersexuality, ${ }^{28}$ one experienced paraesthesiae, and two experienced paraesthesiae and diplopia. One of these latter patients had a perioperative complication, namely a mild subarachnoid haemorrhage, the only presenting symptom being transient diplopia. No permanent lesions occurred in any patient, as confirmed by neurological examination and magnetic resonance imaging.

\section{DISCUSSION}

The results of cognitive assessment in the overall group of 20 patients treated by HFS of the subthalamic nucleus with 12 months of follow up showed that three months after surgery, with stimulators off, there was a highly significant decline in a letter verbal fluency task and a significant decline in a episodic verbal memory task (immediate and delayed recall of the RAVLT), while a significant postoperative improvement was observed on the MMSE and on a task of executive function (the MWCST, with a decreased number of perseverative errors). Six and 12 months after surgery, with stimulators in the on condition, there was a significant postoperative decline in letter verbal fluency (although not as marked as at three months) and an improvement in the MMSE and the MWCST (increased number of correct criteria, decreased number of total and perseverative errors).

In the overall group of 20 patients, there was a significant postoperative decrease in both depressive and anxiety symptoms and an improvement in parkinsonian motor symptoms. Improvements in quality of life and activities of daily living off drug treatment were observed across all postoperative assessments. Similar results were observed in the subgroup of nine patients with an 18 month follow up. Following implantation, 11 patients experienced adverse events, which were persistent in four.

A postoperative decline in a letter verbal fluency task was observed in the overall group of 20 patients across all postoperative sessions. This decline was marginally significant at six and 12 months (with the stimulators in the on condition) and highly significant at three months (with stimulators off). This observation may indicate that a decline in verbal fluency was either more pronounced in the early postoperative stages or that it was attenuated by stimulation (or both). In this respect, it has been reported that the postoperative decline in letter verbal fluency might be more marked at three months than at 12 months, ${ }^{10}$ and that it is attenuated when stimulators are in the on condition. ${ }^{9}$ The latter finding is in agreement with our observation of a significant postoperative decline in letter verbal fluency only at three and 18 months (with stimulators off) in the subgroup of nine patients with an 18 month follow up, but not at six and 12 months (with stimulators on). In all, these findings support the view that a decline in verbal fluency may be attenuated by stimulation of the subthalamic nucleus.

Declines in letter or category fluency have been reported in patients with Parkinson's disease after unilateral or bilateral 
pallidotomy ${ }^{27} 29$ and after surgery for electrode implantation in the internal globus pallidus ${ }^{30}$ or subthalamic nucleus. ${ }^{9}$ As a decline in verbal fluency may also be observed in non-operated patients, this skill could be considered "at risk" in patients with Parkinson's disease. The decline in verbal fluency observed in patients with HFS of the subthalamic nucleus might arise from surgical microlesions of critical neural systems located close to the anterior cingulate cortex in relation with the parasagittal trajectory used for electrode implantation," within the distributed "cognitive" loops involved in verbal fluency tasks. It is also possible that HFS of the subthalamic nucleus might influence the activity of such cognitive loops involved in verbal fluency tasks by attenuating the decline in verbal fluency. According to this hypothesis cognitive deficits induced by surgery could be compensated by stimulation of the subthalamic nucleus. ${ }^{10}$

Our study expands earlier investigations that have assessed cognition in Parkinson's disease only with stimulators turned on. Ardouin et al investigated cognition and mood in 62 patients before and three to six months after bilateral HFS of the subthalamic nucleus or internal globus pallidus with stimulators in the on condition. ${ }^{8}$ That study showed no significant postoperative decline in memory or executive functions, but detected a reduction in letter verbal fluency together with an improvement in mood in both the subthalamic nucleus group and in the whole patient population (which included those with globus pallidus stimulation). Moreover, postoperative improvement was observed in the whole population on the trail making test, a task sensitive to selective visual attention, motor speed, and cognitive flexibility. Saint-Cyr et al investigated neuropsychological changes in a group of 11 patients with Parkinson's disease treated with HFS of the subthalamic nucleus, including six patients older than 69 years. ${ }^{5}$ Assessments were always undertaken with the stimulators in the on condition. At three to six months postoperatively there was a significant decline in tasks of verbal fluency, verbal and visuospatial memory, and executive function (trail making B test). At nine to 12 months, a significant postoperative decline was observed in tasks of verbal fluency, verbal and visuospatial memory, working memory, and executive function (trail making $\mathrm{B}$ test, conditional associative learning test), and in the frontal lobe personality scale. The investigators concluded that bilateral HFS of the subthalamic nucleus can have a negative impact on frontal executive functions, especially in patients older than 69 years, suggesting that further studies are needed to investigate the possible reversibility of these effects when HFS is turned off. Trepanier et al compared the neuropsychological outcome in patients with HFS of the internal globus pallidus or subthalamic nucleus ${ }^{31}$ and suggested that older patients were at risk of cognitive and behavioural decline after bilateral HFS of the subthalamic nucleus, while globus pallidus stimulation may be safer. Alegret and coworkers ${ }^{6}$ assessed cognition and behaviour in 15 patients with Parkinson's disease before and three months after surgery for bilateral HFS of the subthalamic nucleus, with stimulators in the on condition. They observed a postoperative improvement in both the trail making B test and the Maudsley obsessive compulsive inventory. This study, however, showed a postoperative decline in a visuospatial task (the line orientation test), in tasks of verbal memory and verbal fluency, and in the Stroop word-colour test (an attentional task measuring the inhibition of cognitive interference). Dujardin et al investigated nine patients before and at three and 12 months after surgery for HFS of the bilateral subthalamic nucleus, with stimulators on. ${ }^{7}$ They observed a significant postoperative decline in episodic verbal memory only at three months, and a decline in the Stroop word-colour test at 12 months. Behavioural changes (apathy, depression, anxiety) were also reported in four patients.

Recently, two studies have assessed postoperative cognitive performance in patients with Parkinson's disease treated by
HFS with the stimulators in the on versus the off condition. Jahanshahi et al investigated the effects of bilateral HFS on executive functions in seven Parkinson's disease patients with electrode implantation in the subthalamic nucleus and in six with implantation in the internal globus pallidus. ${ }^{9}$ The patients were assessed three times: with stimulators off, with stimulators on, and with stimulators off again. With stimulators on, both the subthalamic nucleus and globus pallidus groups showed a decline in a conditional associative learning task and improved performance on various tests of executive function (trail making tests A and B, paced visual serial addition test, missing digit test, and the naming of colour subtest of the Stroop test). With the stimulators on, the subthalamic nucleus group showed significant improvement on some further tests of executive function (MWCST, random number generation).

In the other study, Pillon et al investigated the effects of subthalamic nucleus or globus pallidus stimulation by comparing cognitive performance of two groups of patients with stimulators on or off at three to 12 months after surgery. ${ }^{10}$ When stimulators were in the on condition, a significant improvement in tests of psychomotor speed and spatial working memory was observed only in the patients with stimulation of the subthalamic nucleus. No cognitive long term effect of HFS was detected 12 months after surgery except for a mild postoperative decline in a semantic category verbal fluency task in the patients with stimulation of the subthalamic nucleus, which was observed at three and 12 months under both conditions (stimulators on or off).

In our study, a postoperative decline was detected in a task of episodic verbal memory (immediate and delayed recall of the RAVLT) at three months (with stimulators off), but not at six and 12 months (with stimulators on). This observation might suggest that, following the subthalamic nucleus implant, a slight decline in episodic verbal memory could be either more evident in the early stages of postoperative follow up or attenuated by subthalamic nucleus stimulation per se, or both. The observation of a postoperative decline in episodic verbal memory at three months ${ }^{6728}$ supports the view that a decline in episodic verbal memory may be more evident in the early postoperative stages. On the other hand, the slight but significant improvement observed in our study on the MMSE at six and 12 months after surgery, with stimulators on, is difficult to interpret, as this task is aimed at assessing the overall cognitive status by screening various major cognitive domains. Such an improvement on the MMSE could reflect either a small but genuine postoperative improvement in overall cognitive functioning induced by subthalamic nucleus stimulation, or (more likely) a practice effect, or both.

Similarly, the improved postoperative performance detected in the overall group of our 20 patients on the MWCST in all postoperative assessments (but more notably at six and 12 months, with stimulators on) could arise either from a genuine improvement in specific frontal executive functions (set shifting ability) or (perhaps more likely) from a practice effect, or both. This finding, however, is in agreement with some previous reports of improved performance with stimulators on detected on various tests of executive function, such as the MWCST $^{9}$ and tasks of spatial working memory, ${ }^{10}$ suggesting that stimulation of the subthalamic nucleus could, at least in some patients, improve executive functions and working memory. On the other hand, it should be pointed out that these findings are at variance with the results of the study conducted by Saint-Cyr et al, ${ }^{5}$ which suggests that bilateral subthalamic nucleus HFS can have a negative impact on frontal executive functions, especially in patients older than 69 years. It has been proposed ${ }^{31}$ that differences between the neuropsychological outcome in patients with Parkinson's disease operated on in different centres may be influenced by various factors, including the location and extent of the neurosurgical lesions and the characteristics of the patients 
selected for surgery (age, preoperative cognitive status, comorbidity with other conditions such as psychiatric disorders). In this respect, it is worth commenting that the mean age of the patients in Saint-Cyr's study (66.5 years) was about nine years older than the mean age of the patients in our study (57.0 years), who showed no postoperative impairment in tasks of executive function.

The marked improvement in parkinsonian motor symptoms observed in the present series might at least partially explain not only the postoperative improvement in the quality of life and activities of daily living but also the postoperative decrease in depressive and anxiety symptoms-as a psychological response to the alleviation of disabling motor symptoms. ${ }^{9}$ However, the possible influence of HSF of the subthalamic nucleus on the neural circuits involved in mood should also be considered. ${ }^{428} 32$

The analysis of postoperative changes in individual patients in cognitive, behavioural, and functional variables showed some individual variability which could, at least in part, reflect slight variability in lead placement. ${ }^{33}$

\section{Limitations}

Longitudinal studies on the neuropsychological outcome after surgical treatments of Parkinson's disease raise several methodological issues. First, it is necessary to consider that cognitive decline may occur in patients with Parkinson's disease over time, although very little decline is usually observed over a 12 month period. ${ }^{5}$ Second, we have already been mentioned that in serial neuropsychological assessments improved performance may result from practice effects, especially with short test-retest intervals. ${ }^{11}$ To mitigate such practice effects, various approaches could be employed, such as the use of parallel forms of cognitive tasks or the comparison of results with an unoperated group of patients. However, the value of these approaches is restricted by the limited availability of parallel forms and by the logistic problems incurred in the recruitment of an unoperated group of patients. ${ }^{34}$ Given the limited availability of alternative forms for many of the cognitive tasks selected for our study, relatively long intervals between cognitive assessments under different stimulation conditions were chosen to limit practice effects.

An additional methodological issue derives from the choice of the most appropriate pharmacological conditions during preoperative and postoperative cognitive testing. Neuropsychological assessments in Parkinson's disease patients treated by HFS of the subthalamic nucleus have been carried out under variable pharmacological conditions. In some studies, both preoperative and postoperative assessments were performed with the patients on anti-parkinsonian drug treatment. ${ }^{67}$ In other studies, preoperative and postoperative assessments were carried out without anti-parkinsonian drugs in all patients, ${ }^{69}$ or in most of them. ${ }^{810}$ In one study, conducted in patients treated by unilateral internal globus pallidus HFS, ${ }^{35}$ subjects were assessed with medication preoperatively and without medication postoperatively. It is not possible to exclude the possibility that a postoperative reduction in anti-parkinsonian drugs in patients treated by HFS of the subthalamic nucleus could to some extent influence their performance on cognitive tasks. Although cognitive assessments in patients free of antiparkinsonian drugs for at least 12 hours before and after surgery could minimise the influence of changes in drugs on cognitive performance, it should be pointed out that in at least some patients the severity of the motor symptoms while they are off drug treatment (particularly preoperatively) might not permit cognitive testing. ${ }^{6}$

\section{Conclusions}

Our study supports the view that bilateral HFS of the subthalamic nucleus is a relatively safe procedure with regard to cognitive and behavioural morbidity over a long term follow up (up to 18 months). However, individual variability in postoperative cognitive and behavioural outcome-as observed in our sample and in other studies-invites caution. Further investigations are necessary to elucidate the reasons for such individual variability in outcome, which could be influenced by various factors such as the characteristics of the patients (age, preoperative cognitive status, history of psychiatric disorders) and the exact location of the electrode placement. In addition, our findings indicate that stimulation of the subthalamic nucleus per se does not seem to impair cognitive performance in patients with Parkinson's disease, suggesting-in agreement with earlier results ${ }^{9}$ - that subthalamic stimulation could attenuate the observed postoperative decline in verbal fluency tasks, at least in some patients.

Authors' affiliations

A Daniele, A Albanese, M F Contarino, P Zinzi, A Barbier, F Gasparini, L M A Romito, A R Bentivoglio, Istituto di Neurologia, Università Cattolica, Rome, Italy

M Scerrati, Istituto di Neurochirurgia, Università Cattolica

Competing interests: none declared

\section{REFERENCES}

1 Krack P, Pollak $P$, Limousin $P$, et al. Subthalamic nucleus or internal pallidal stimulation in young onset Parkinson's disease. Brain 1998; 121:451-7

2 Kumar R, Lozano AM, Sime E, et al. Comparative effects of unilateral and bilateral subthalamic nucleus deep brain stimulation. Neurology 1999:53:561-6.

3 Romito LM, Scerrati M, Contarino MF, et al. Long-term follow up of subthalamic nucleus stimulation in Parkinson's disease. Neurology 2002;58: 1546-50.

4 Kumar R, Krack P, Pollak P. Transient acute depression induced by high-frequency deep-brain stimulation. N Engl J Med 1999;341:1003-4

5 Saint-Cyr JA, Trepanier LL, Kumar R, et al. Neuropsychological consequences of chronic bilateral stimulation of the subthalamic nucleus in Parkinson's disease. Brain 2000;1 23:2091-108.

6 Alegret $M$, Junque $C$, Valldeoriola $F$, et al. Effects of bilateral subthalamic stimulation on cognitive function in Parkinson disease. Arch Neurol 2001;58:1223-7

7 Dujardin K, Defebvre L, Krystkowiak P, et al. Influence of chronic bilateral stimulation of the subthalamic nucleus on cognitive function in Parkinson's disease. J Neurol 2001:248:603-11.

8 Ardouin C, Pillon B, Peiffer E, et al. Bilateral subthalamic or pallidal stimulation for Parkinson's disease affects neither memory nor executive functions: a consecutive series of 62 patients. Ann Neurol 1999:46:217-23.

9 Jahanshahi M, Ardouin CM, Brown RG, et al. The impact of deep brain stimulation on executive function in Parkinson's disease. Brain 2000; 1 23: 1 1 42-54.

10 Pillon B, Ardouin C, Damier P, et al. Neuropsychological changes between "off" and "on" STN or GPi stimulation in Parkinson's disease. Neurology 2000;55:411-18.

11 Lezak MD. Neuropsychological assessment. New York: Oxford University Press, 1995

12 Moro E, Scerrati M, Romito LM, et al. Chronic subthalamic nucleus stimulation reduces medication requirements in Parkinson's disease. Neurology 1999:53:85-90.

13 Hughes AJ, Daniel SE, Kilford L, et al. Accuracy of clinical diagnosis of idiopathic Parkinson's disease: a clinico-pathological study of 100 cases. J Neurol Neurosurg Psychiatry 1992;55:181-4.

14 Langston JW, Widner H, Goetz CG, et al. Core assessment program for intracerebral transplantations (CAPIT). Mov Disord 1992;7:2-13.

15 American Psychiatric Association. Diagnostic and statistical manual of mental disorders. Washington: American Psychiatric Association, 1994.

16 Fahn S, Elton RL, Members of the UPDRS Development Committee. Unified Parkinson's disease rating scale. In: Fahn S, Marsden CD, Calne $D$, et al. Recent developments in Parkinson's disease. Florham Park: Macmillan Healthcare Information 1987: 153-63

17 Folstein MF, Folstein SE, McHugh PR. "Mini-mental state". A practica method for grading the cognitive state of patients for the clinician. $J$ Psychiatr Res 1975;12:189-98.

18 Wechsler D. The measurement and appraisal of adult intelligence. Baltimore: The Psychological Corporation, 1958

19 Milner B. Interhemispheric differences in the localization of psychological processes in man. Br Med Bull 1971;27:272-7.

20 Rey A. Mémorisation d'une séries de 15 mots en 5 répetitions. In: Rey A ed. L'examen clinique en psychologie. Paris: Presses Universitaires de France, 1958.

21 Raven JC. Progressive matrices (1947), sets A, Ab, B: board and book forms. London: 1949.

22 Nelson HE. A modified card sorting test sensitive to frontal lobe defects. Cortex 1976;12:313-24. 
23 Borkowski JG, Benton AL, Spreen O. Word fluency and brain damage. Neuropsychologia 1967;140.

24 Zung WW. Self-rating depression scale in an outpatient clinic. Further validation of the SDS, 1965

25 Zung WW. How normal is anxiety? Current concepts (report). Kalamazoo, MI: Upjohn Company, 1980

26 de Boer AG, Wijker W, Speelman JD, et al. Quality of life in patients with Parkinson's disease: development of a questionnaire. J Neurol Neurosurg Psychiatry 1996:61:70-4.

27 Trepanier LL, Saint-Cyr JA, Lozano AM, et al. Neuropsychological consequences of posteroventral pallidotomy for the treatment of Parkinson's disease. Neurology 1998;51:207-15.

28 Romito LM, Raja M, Daniele A, et al. Transient mania with hypersexuality following surgery for high frequency stimulation of the subthalamic nucleus in Parkinson's disease. Move Disord 2002;19:1371-4

29 Scott R, Gregory R, Hines N, et al. Neuropsychological, neurological and functional outcome following pallidotomy for Parkinson's disease. A consecutive series of eight simultaneous bilateral and twelve unilateral procedures. Brain 1998;121:659-75.

30 Troster AI, Fields JA, Wilkinson SB, et al. Unilateral pallidal stimulation for Parkinson's disease: neurobehavioral functioning before and 3 months after electrode implantation. Neurology 1997;49:1078-83.

31 Trepanier LL, Kumar R, Lozano AM, et al. Neuropsychological outcome of GPi pallidotomy and GPi or STN deep brain stimulation in Parkinson's disease. Brain Cogn 2000:42:324-47.

32 Beiiani BP, Damier $\mathrm{P}$, Arnulf $\mathrm{I}$, et al. Transient acute depression induced by high-frequency deep-brain stimulation. N Engl J Med 1999;340:1476-80.

33 Kumar $\mathbf{R}$, Lang AE, Rodriguez-Oroz MC, et al. Deep brain stimulation of the globus pallidus pars interna in advanced Parkinson's disease. Neurology 2000;55:S34-9

34 York MK, Levin HS, Grossman RG, et al. Neuropsychological outcome following unilateral pallidotomy. Brain 1999:122:2209-20.

35 Vingerhoets $\mathbf{G}$, van der LC, Lannoo $E$, et al. Cognitive outcome after unilateral pallidal stimulation in Parkinson's disease. I Neurol Neurosurg Psychiatry 1999;66:297-304

\section{Look before you leap if liable to pressure palsies}

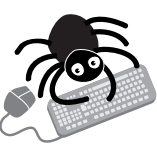

Please visit the Journal of Neurology, Neurosurgery, and Psychiatry website [www jnnp.com] for link to this full article.
Deple with hereditary neuropathy with liability to pressure palsies (HNPP) should be warned against sports like parachuting and rock climbing because of their increased risk of severe nerve injury from tight harnesses.

An unusual case report describes how an 18 year old woman with no history of HNPP experienced lasting weakness in both arms during her first parachute jump. She was harnessed by tight shoulder straps to an instructor, and during the jump felt pain in her left shoulder. On landing she could not move either arm or get up. About $80 \%$ strength returned to her right arm after 5-10 minutes but her left remained numb and weak.

A week later she had no pain in her neck, back, or legs or weakness or tingling but still had mild weakness and slow reflexes in the right arm and severe weakness and no reflexes in the left. This suggested cervical central cord syndrome, but an MRI scan showed no abnormalities. Nerve conduction studies and electromyography two weeks after the event showed sensorimotor neuropathy in the arms and legs and prolonged latency of conduction at classic compression sites but no active denervation. Fluorescent in situ hybridisation disclosed a deletion on chromosome 17pl1.2 characteristic of HNPP. Recovery took nine months.

Involvement of nerves in both arms is unusual and probably arose from the tight harness. Patients with HNPP suffer recurring nerve injury after the mildest compression forces, and carrying heavy weights over their shoulders, resting on their elbows, or crossing their legs is discouraged.

A British Journal of Sports Medicine 2002;36:465-466. 